\title{
Lumbar Facet Cyst Causing Incomplete Cauda Equina Syndrome: A Case Report and Review of Literature
}

\author{
Manoj Ramachandraiah ${ }^{1}$, Thomas J Kishen ${ }^{2}$
}

\begin{abstract}
Background: Intraspinal facet cyst or juxta-facet cyst is a term that includes synovial and ganglion cysts in the spine. Facetal synovial cysts are fluid-containing synovial outpouchings arising from degenerated facet joints resulting from chronic hypermobility of the joints. These cysts can cause low back pain, radicular leg pain, and in rare cases cauda equina syndrome.

Case description: In this report, we would like to present the occurrence of incomplete cauda equine syndrome resulting from a facet synovial cyst in a 65-year-old woman who presented with weakness in both lower limbs, difficulty in walking, and saddle anesthesia. The symptoms resolved following a posterior decompression, cyst excision, and instrumented fusion surgery.

Conclusion: This report highlights the occurrence of acute cauda equina syndrome in a 65-year-old woman resulting from an L4-L5 facet synovial cyst. Prompt diagnosis and immediate surgical intervention in the form of decompression and fusion surgery lead to a favorable outcome.

Clinical message: Acute cauda equina syndrome in a patient with a lumbar facet cyst is a relatively uncommon presentation. It requires early diagnosis and immediate surgical intervention in the form of decompression to prevent neurological deterioration and to have a favorable outcome.

Keywords: Cauda equina syndrome, Decompression, Facetal cyst

Journal of Orthopedics and Joint Surgery (2021): 10.5005/jp-journals-10079-1033
\end{abstract}

\section{BACKGROUND}

Intraspinal facet cyst or juxta-facet cyst is a term that includes synovial and ganglion cysts in the spine. ${ }^{1}$ Facetal synovial cysts are fluid-containing synovial outpouchings (and hence lined by synovial tissue) arising from degenerated facet joints resulting from chronic hypermobility of the joints. ${ }^{2,3}$ Ganglion cysts, on the other hand, contain a more viscous fluid and lack a synovial lining. ${ }^{2,3}$ Despite the histological distinction between synovial and ganglion cysts, the clinical presentation, management, and prognosis are similar. ${ }^{4}$ Eyster and Scott reported a $0.6 \%$ incidence of lumbar synovial cysts based on a study of 1,800 CT/MRI scans. ${ }^{5}$ These cysts, seen more commonly in the mobile lower lumbar spine, ${ }^{6}$ can cause low back pain, radicular leg pain, and in rare cases cauda equina syndrome. . $^{3,6-8}$

Cauda equina syndrome may occur due to hemorrhage into the cyst $^{2,4}$ or rapid expansion of a cyst. ${ }^{9}$ In this report, we would like to present the occurrence of incomplete cauda equine syndrome resulting from a facet synovial cyst in a 65 -year-old woman who presented with weakness in both lower limbs, difficulty in walking, and saddle anesthesia. The symptoms resolved following a posterior decompression, cyst excision, and instrumented fusion surgery.

\section{Case Description}

A 65-year-old woman presented with weakness, heaviness, and numbness of both lower limbs along with perianal numbness of 10 days of duration resulting in an inability to walk unassisted. However, she did not report any back/leg pain or difficulty with bladder or bowel function. Neurological examination of the lower limbs revealed bilateral ankle dorsiflexion power (4/5), bilateral extensor hallucis longus (2/5), bilateral hip abduction (3/5), bilateral ankle plantar flexion (3/5), bilateral knee flexion $(4 / 5)$ with reduced pinprick, and light touch sensation over the \begin{tabular}{l}
\hline 1,2 Spine Care Centre, Manipal Hospitals, Bengaluru, Karnataka, India \\
Corresponding Author: Thomas J Kishen, Spine Care Centre, Manipal \\
Hospital, Bengaluru, Karnataka, India, Phone: +91 8025023334, e-mail: \\
thomas.kishen@manipalhospitals.com \\
How to cite this article: Ramachandraiah M, Kishen TJ. Lumbar Facet \\
Cyst Causing Incomplete Cauda Equina Syndrome: A Case Report and
\end{tabular} Review of Literature. J Orth Joint Surg 2021;3(1):60-62.

Source of support: Nil

Conflict of interest: None

$\mathrm{L} 5, \mathrm{S1}, \mathrm{S} 2$, and perianal region bilaterally. There was no evidence of upper motor neuron signs and the peripheral pulses were well felt in both lower limbs.

Radiographs revealed a grade I degenerative spondylolisthesis at the L4-L5 level (Fig. 1) and the MRI scan (Fig. 2) showed a posterior midline epidural cyst below the ligamentum flavum causing cauda equine compression at the L4-L5 level. The cyst revealed isointense signals compared with cerebrospinal fluid on T2-weighted images and hypointense signals on T1 images which supported its nonhemorrhagic content.

Because of the significant motor and sensory deficits, surgery was considered in the form of decompression and instrumented fusion. After a thorough preoperative evaluation, surgery was performed in the form of an L4 laminectomy and decompression and L4-L5 posterolateral instrumented fusion. The cyst, identified below the ligamentum flavum and communicating with the left L4-L5 facet joint was excised along the left L4-L5 facet joint and the dural sac and nerve roots were decompressed followed by an L4-L5 posterolateral instrumented fusion (Fig. 3). Histopathological examination of the excised cyst wall revealed a single layer of flattened synoviocytes and mild inflammatory infiltrate along with 
focal areas of calcification and cartilage fragments consistent with a diagnosis of the synovial cyst (Fig. 4).

The postoperative period was uneventful and rapid neurological improvement was noted. The motor power and sensations returned to normal by the 3 months mark and the patient was ambulating independently and doing well at the last follow-up visit (18 months post-surgery).

\section{Discussion}

Kao et al. classified extradural intraspinal cysts into three groups(1) Perineural cysts (arising from dorsal root ganglion), (2) Arachnoid cysts (pedicle attachment to spinal dura near nerve root), and (3) Juxta-facet cysts-ganglion and synovial cysts attached to the periarticular connective tissue of facet joints. ${ }^{1}$ Ganglion and synovial cysts are also referred to as intraspinal facet cysts ${ }^{1,3}$ or cystic malformations of mobile spine (CYFMOS) to encompass its heterogeneity. ${ }^{7}$ Various hypotheses have been proposed regarding

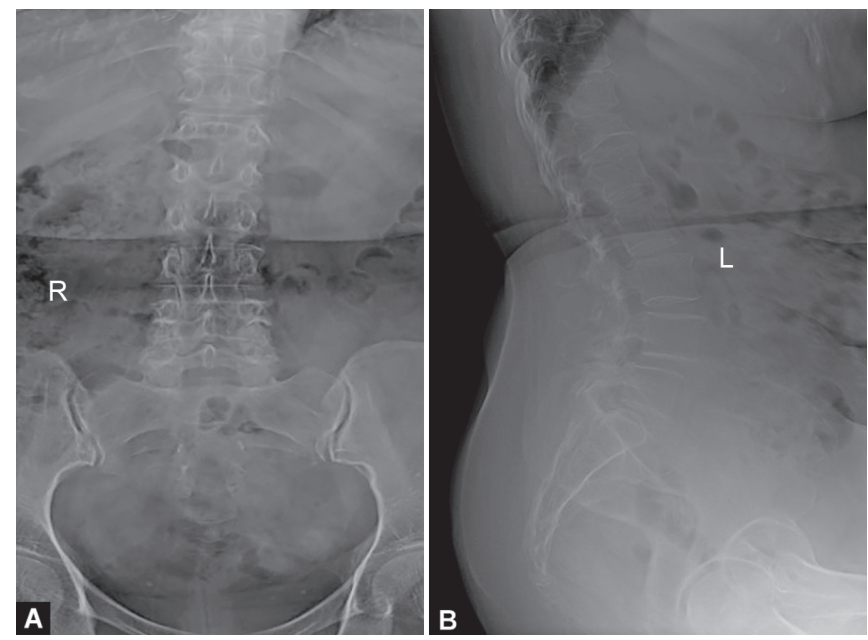

Figs 1 A and B: Anteroposterior (A) and lateral (B) preoperative radiographs showing grade I spondylolisthesis at the L4-L5 level the development of these cysts including synovial herniation through a defective capsule, periarticular fibrous tissue undergoing mucinous degeneration, and pluripotent mesenchymal cells in ligamentum flavum undergoing proliferation and focal hemorrhage due to repetitive microtrauma. ${ }^{2,8}$ Despite the histological distinction between synovial and ganglion cysts, the clinical presentation, management, and prognosis are similar. ${ }^{4}$

The incidence of synovial cysts is $0.6 \%$ based on a study of 1,800 CT and MRI scans ${ }^{5}$ being more common in the lumbar spine $(94 \%)$ and a majority occurring at the L4-L5 level. ${ }^{10}$ These cysts typically present after the age of 50 years, ${ }^{3,6,11}$ with facet arthropathy noted in $90 \%{ }^{12}$ and spondylolisthesis in $40 \%$ of patients. ${ }^{12}$ While a majority present with unilateral or bilateral radiculopathy (55-97\%), 25-44\% present with neurological claudication, $26-40 \%$ present with motor deficits, and $13 \%$ present with cauda equine syndrome. ${ }^{3,6-8}$ Facet cysts can cause cauda equine syndrome due to hemorrhage within synovial cyst or rapidly expanding cysts. ${ }^{2,4,9}$

Nonsurgical management in the form of rest, analgesics, and spinal exercises and epidural steroids have been generally unsatisfactory, ${ }^{3,6,13}$ although there is a few reports of spontaneous regression of facet cysts. ${ }^{14-16}$ Percutaneous rupture of the cyst has been shown to have a high recurrence rate. ${ }^{17}$ In the presence of recalcitrant pain and neurological deficits, surgical intervention is an effective option. ${ }^{11}$ The recurrence rate following a cyst excision/ decompression only procedure is $11 \%$ with better outcomes following cyst excision/decompression and fusion in the presence of spondylolisthesis. ${ }^{3,6,7,11}$ Adhesions between the cyst and underlying dura have been reported, ${ }^{6}$ requiring meticulous dissection to avoid a dural tear.

The patient discussed in this report presented with weakness and numbness in both lower limbs and perianal numbness without frank urinary retention. These symptoms could fall under the description of incomplete cauda equina syndrome. ${ }^{18}$ The isointense signals similar to CSF in our facet cyst ruled out the presence of hemorrhage into the cyst. Because of the significant neurological deficits and radiological evidence of spondylolisthesis, a decompression and instrumented fusion was planned. The

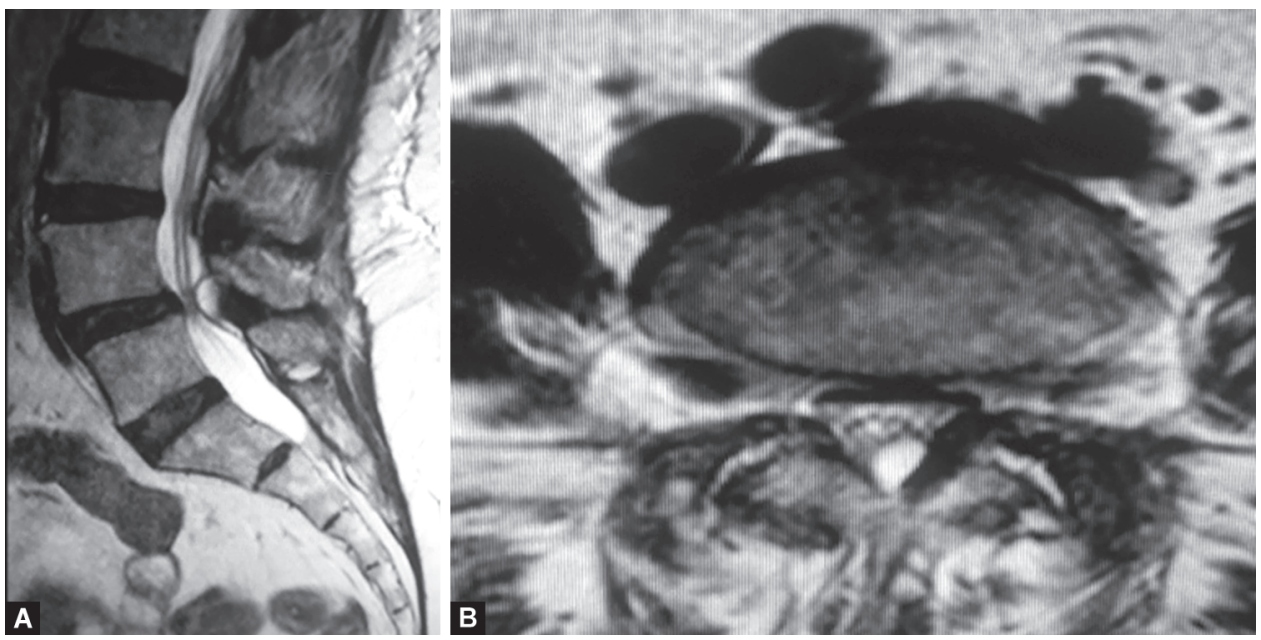

Figs 2A and B: Sagittal (A) and axial (B) T2W MRI images showing posterior midline epidural cyst below the ligamentum flavum causing cauda equine compression at the L4-L5 leve 

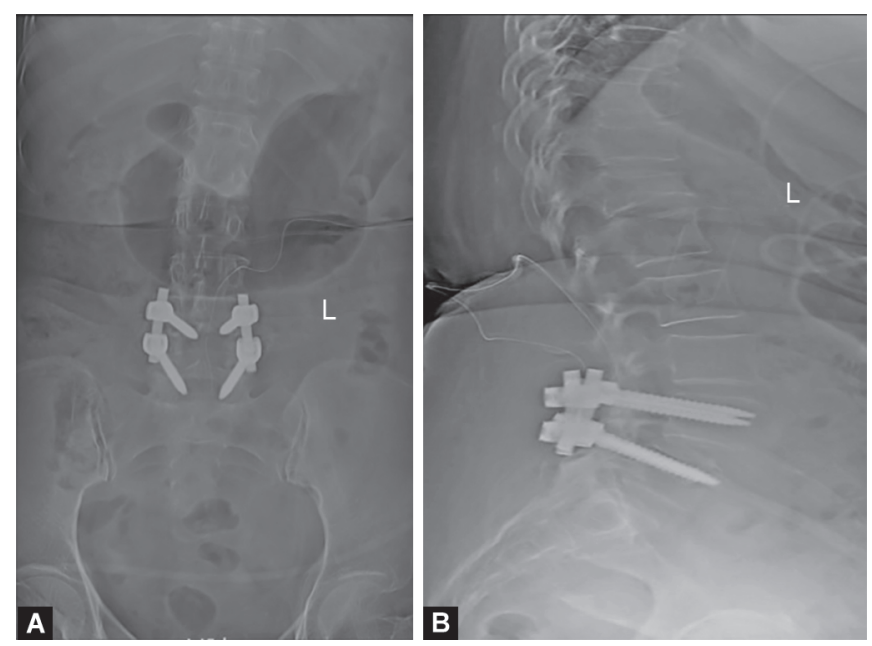

Figs $3 \mathrm{~A}$ and B: Anteroposterior (A) and lateral (B) postoperative radiographs after $\mathrm{L} 4-\mathrm{L} 5$ posterior decompression, cyst excision, and posterolateral instrumented fusion

patient withstood the procedure well with complete resolution of neurological deficits within 3 months.

\section{ConcLusion}

This report highlights the occurrence of acute cauda equina syndrome in a 65-year-old woman resulting from an L4-L5 facet synovial cyst. Prompt diagnosis and immediate surgical intervention in the form of decompression and fusion surgery lead to a favorable outcome.

\section{Clinical Message}

Acute cauda equina syndrome in a patient with a lumbar facet cyst is a relatively uncommon presentation. It requires early diagnosis and immediate surgical intervention in the form of decompression to prevent neurological deterioration and to have a favorable outcome.

\section{References}

1. Kao C, Winkler S, Turner J. Synovial cyst of spinal facet. J Neurosurg 1974;41(3):372-376. DOI: 10.3171/jns.1974.41.3.0372.

2. Tatter $S$, Cosgrove $G$. Hemorrhage into a lumbar synovial cyst causing an acute cauda equina syndrome. J Neurosurg 1994;81(3):449-452. DOI: 10.3171/jns.1994.81.3.0449.

3. Hsu K, Zucherman J, Shea W, et al. Lumbar intraspinal synovial and ganglion cysts (facet cysts). Spine 1995;20(1):80-89. DOI: 10.1097/00007632-199501000-00015.

4. Machino M, Yukawa $Y$, Ito $K$, et al. Spontaneous hemorrhage in an upper lumbar synovial cyst causing subacute cauda equina syndrome. Orthopedics 2012;35(9):e1457-e1460. DOI: 10.3928/01477447-20120822-41.

5. Eyster E, Scott W. Lumbar synovial cysts: report of eleven cases. Neurosurgery 1989;24(1):112-115. DOI: 10.1227/00006123-19890100000021.

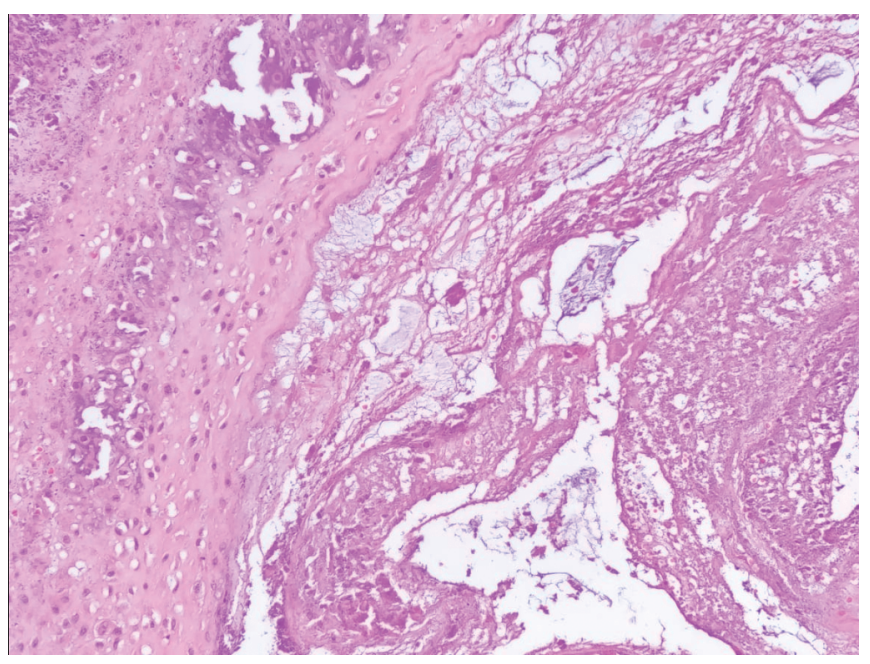

Fig. 4: H\&E-stained specimen (20× magnification) of the excised cyst wall showing a single layer of flattened synoviocytes and mild inflammatory infiltrate along with focal areas of calcification and cartilage fragments

6. Epstein N. Lumbar synovial cysts. J Spinal Disord Tech 2004;17(4):321325. DOI: 10.1097/01.bsd.0000096267.75190.eb.

7. Christophis P, Asamoto S, Kuchelmeister K, et al. "Juxtafacet cysts", a misleading name for cystic formations of mobile spine (CYFMOS). Eur Spine J 2007;16(9):1499-1505. DOI: 10.1007/s00586-006-0287-5.

8. Muir JJ, Pingree MJ, Moeschler SM. Acute cauda equina syndrome secondary to a lumbar synovial cyst. Pain Physic 2012;15(5):435-440. DOI: 10.36076/ppj.2012/15/435.

9. lacob G, Rotund B. Lumbar L4-L5 ganglion cyst with hemicauda equina syndrome. Report of a case and review of the literature. Roman Neurosurg 2013;20(4):335-343. DOI: 10.2478/romneu-20130017.

10. Howington J, Connolly E, Voorhies R. Intraspinal synovial cysts: 10-year experience at the Ochsner clinic. J Neurosurg: Spine 1999;91(2):193-199.

11. Khan A, Synnot K, Cammisa F, et al. Lumbar synovial cysts of the spine. J Spinal Disord Tech 2005;18(2):127-131. DOI: 10.1097/01. bsd.0000156830.68431.70.

12. Banning $C$, Thorell W, Leibrock L. Patient outcome after resection of lumbar juxtafacet cysts. Spine 2001;26(8):969-972. DOI: 10.1097/00007632-200104150-00024.

13. Shah RV, Lutz GE. Lumbar intraspinal synovial cysts: conservative management and review of the world's literature. Spine J 2003;3(6):479-488. DOI: 10.1016/S1529-9430(03)00148-7.

14. Houten J, Sanderson S, Cooper P. Spontaneous regression of symptomatic lumbar synovial cysts. J Neurosurg: Spine 2003;99(2):235-238. DOI: 10.3171/spi.2003.99.2.0235.

15. Maezawa $Y$, Baba $H$, Uchida $K$, et al. Spontaneous remission of a solitary intraspinal synovial cyst of the lumbar spine. Eur Spine J 2000;9(1):85-87. DOI: 10.1007/s005860050016.

16. Swartz PG, Murtagh FR. Spontaneous resolution of an intraspinal synovial cyst. AJNR Am J Neuroradiol 2003;24(6):1261-1263.

17. Martha JF, Swaim B, Wang DA, et al. Outcome of percutaneous rupture of lumbar synovial cysts: a case series of 101 patients. Spine J 2009;9(11):899-904. DOI: 10.1016/j.spinee.2009.06.010.

18. Gleave J, MacFarlane R. Cauda equina syndrome: what is the relationship between timing of surgery and outcome. $\mathrm{Br} J$ Neurosurg 2002;16(4):325-328. DOI: 10.1080/0268869021000032887. 